\title{
Z-99 Empirical inversion of HEM data for sea ice thickness mapping
}

AUTHORS

A. PFAFFLING ${ }^{1}$, C. HAAS ${ }^{1}$ and J. E. REID ${ }^{2}$

Address

${ }^{1}$ Alfred Wegener Institute for Polar and Marine Research, Division Climate Physics, PO Box 12 0161, 27515

Bremerhaven, Germany

${ }^{2}$ University of Tasmania, School of Earth Sciences, Private Bag 79, Hobart, Tasmania 7001, Australia

\section{Summary}

Since 2001, the Alfred Wegener Institute for Polar and Marine Research in Bremerhaven (AWI) has operated a purpose built, unique HEM system to map sea ice thickness in the Arctic and Antarctic. To determine the sea ice thickness from the measured EM field we use an empirical curve fitting approach. The master curve is tuned for every flight leg using measurements over open water between the ice floes in leads or polynyas. The level ice thickness, which is a key value for sea ice science and climate research can be derived with an accuracy of a few centimeters.

\section{Introduction}

For more than ten years the AWI has conducted sea ice thickness measurements to study changes in the thickness distribution and their link to the climate both in the Arctic and the Antarctic. Apart from drilling, the only operational geophysical instrument for that purpose had been a Geonics EM31 dragged along the ice surface.

Frequency domain helicopter electromagnetics (HEM) was introduced to sea ice science in the late 1980s (Kovacs and Holladay, 1990). Being saline, sea water is a good conductor (2.5 $\mathrm{S} / \mathrm{m}$ ) and therefore provides a strong HEM response. Sea ice, however, has a low conductivity around $0.01 \mathrm{~S} / \mathrm{m}$, and the measured EM response depends mainly on height of the system above the seawater. It is thus possible to explore ice thickness with an airborne EM instrument.

Commercially-available HEM devices with lengths from 7 to $10 \mathrm{~m}$ weighing up to $300 \mathrm{~kg}$ are not practical to operate from research icebreakers. Hence the necessity for a new, extremely small and light sensor arose. A fully digital bird operating at two frequencies was developed by the AWI, Ferra Dynamics, Dr. K-P Sengpiel and Aerodata Systems (Fig. 1). The bird is equipped with a laser altimeter to measure the elevation of the sea ice surface and to determine surface roughness, i.e. pressure ridge heights and distribution. A real time laser altimeter display for the pilot allows to fly the bird at 10 to $15 \mathrm{~m}$ height above the ice surface.

\begin{tabular}{|lll|}
\hline Length / weight & $3.4 \mathrm{~m} / 103 \mathrm{~kg}$ \\
Frequency & $3.68 \mathrm{kHz}$ & $112 \mathrm{kHz}$ \\
Coil separation & $2.77 \mathrm{~m}$ & $2.05 \mathrm{~m}$ \\
Coil geometry & Horizontal coplanar \\
Sampling frequency & $10 \mathrm{~Hz}$ (Laser: $100 \mathrm{~Hz})$ \\
Towing cable length & $20 \mathrm{~m}$ \\
Operation altitude & $10-15 \mathrm{~m}$ above ice surface \\
Operation speed & $60-80 \mathrm{knots}(30-40 \mathrm{~m} / \mathrm{s})$ \\
\hline
\end{tabular}

Near Surface 2004 - 10th European Meeting of Environmental and Engineering Geophysics, Utrecht, The Netherlands 
As reducing the coil separation strongly decreases the signal/noise ratio it is a technical challenge to build such a small bird. To reduce the mechanical noise the electronics and coils are mounted on a rigid kevlar plate, which is mechanically decoupled from the bird's shell and towing interface. The main noise-prevention measure is the introduction of a fully digital bird system. All tasks of the bird like signal generation, acquisition and preprocessing as well as calibration, phasing or nulling are managed by a PC inside the bird. The built-in calibration coils allow system assessments once the bird is taken to high altitude for drift evaluation.

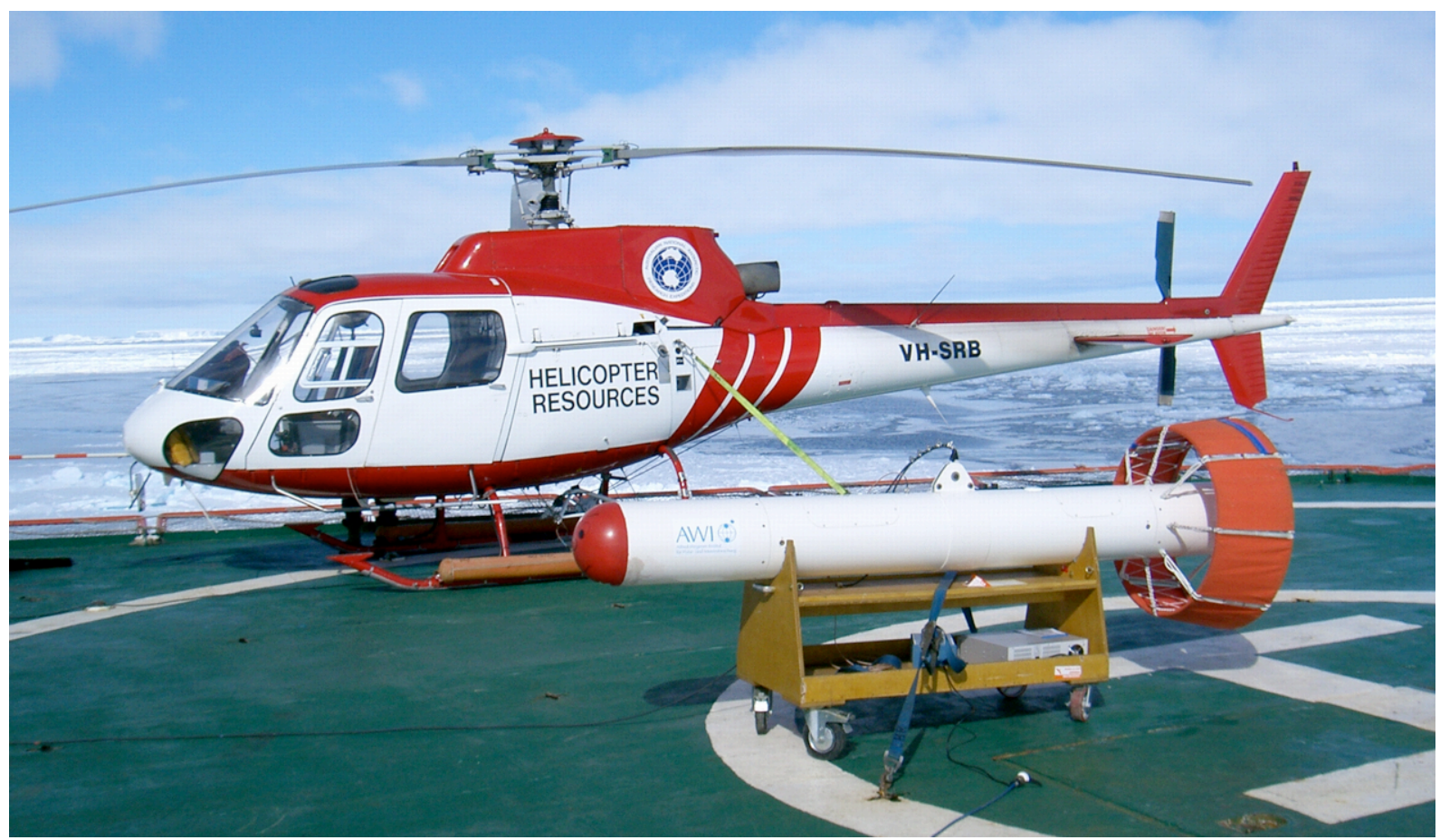

Figure 1: AWI's small sized HEM bird on RSV “Aurora Australis” in Antarctic pack ice.

Calibration and phasing are carried out at the beginning and end of every flight leg. However, system drift in phase and gain has been almost negligible so far. System operation and data transmission to the operator laptop inside the helicopter is performed by a radio ethernet connection. Due to this set-up the bird can be operated from any aircraft equipped with a load hook and a 28 Volt power supply.

\section{Geophysics of sea ice}

Sea ice is a dynamic system floating on the polar oceans exposed to wind and ocean currents. It is composed of level ice floes merged by pressure ridges as well as separated by cracks, leads or several hundred meter wide polynyas. Antarctic pressure ridges can grow up to $8 \mathrm{~m}$ thickness and extend over $10 \mathrm{~m}$ width. However, the thermodynamic history of the sea ice is represented by the level ice thickness, hardly growing any thicker than 3 meter in calm conditions, while ridges and leads are linked to short term deformation events related to winds and currents. While EM sounding yields accurate estimates of level ice thickness, ridge distributions can be retrieved from the laser altimeter data.. The sea ice thickness distribution, expressed as probability density functions, usually shows a main peak representing the level ice thickness. Furthermore it contains estimates of the open water fraction and pressure ridge occurrence and therefore characterizes the local ice regime.

\section{The empirical approach}

The measured normalized secondary field $\left(Z=H_{s} / H_{p}\right.$ [ppm], with $H_{s} \ldots$ secondary magnetic field \& $H_{p} \ldots$ primary magnetic field) is a nonlinear function of the coil separation $r$, sensor 
altitude $h$, transmitter frequency $f$ and the EM properties of the halfspace, namely conductivity $\sigma$ and susceptibility $\mu$ (Equation 1).

$$
Z=r^{3} \int_{0}^{\infty} \frac{\lambda-\sqrt{\lambda^{2}+i \varpi \sigma \mu}}{\lambda+\sqrt{\lambda^{2}+i \varpi \sigma \mu}} \cdot e^{-2 \lambda h} \cdot \lambda^{2} \cdot J_{0}(\lambda r) d \lambda
$$

Assuming the ocean to be a homogeneous halfspace, for given $r$ and $f$ as well as constant $\sigma$ and $\mu, Z$ depends only on $h$. We approximate equation (1) to

$$
Z=C_{1}+C_{2} \cdot e^{C_{3} \cdot h} .
$$

Equation (2)

The parameters $C_{1,2,3}$ are estimated via exponential curve fitting used on measured $Z$ and $h$ over open water. Once the algorithm is tuned, the total thickness $t t$ (= snow + ice thickness) is determined by

$$
t t=h_{E M}-h_{L A} .
$$

$h_{E M}$ is the distance between bird and water surface determined from the measured $Z$ using equation (2), while $h_{L A}$ is the bird altitude over the ice or snow surface measured by the laser. Fig. 2 shows a cross plot of $Z$ over h from a $25 \mathrm{~km}$ long transect over Antarctic pack ice. The measurements over open water can be clearly distinguished as they show the expected exponential behavior with $h$. The fitted exponential curve is also shown on the graph as a solid line. The fit was computed using the points marked as "Sample".

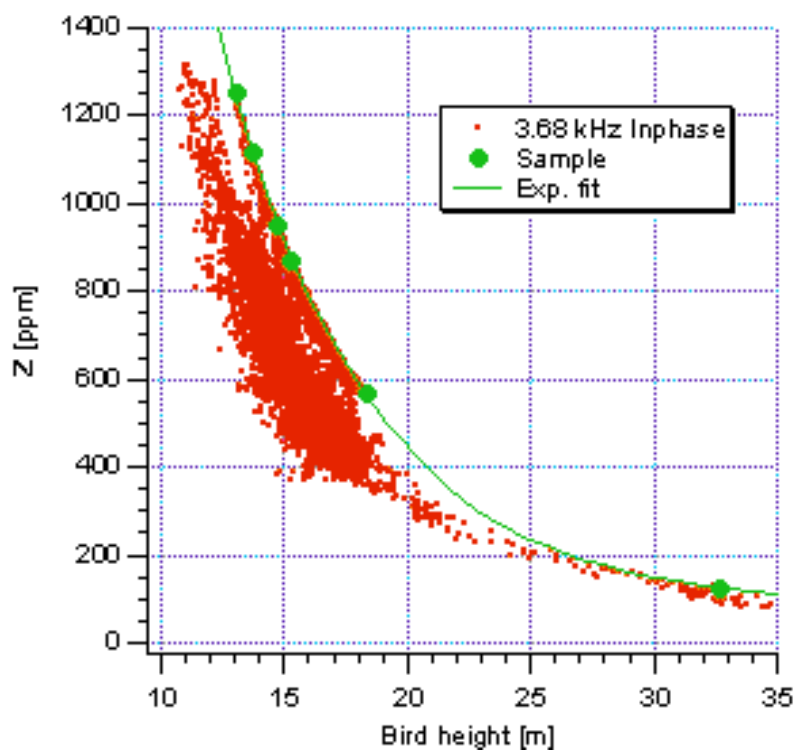

Figure 2: Crossplot of the inphase response at $3.68 \mathrm{kHz}$ and the bird height measured by the laser altimeter. Small dots show field data. The line represents the exponential fitting curve.

\section{Validation of the algorithm}

During an expedition to Antarctica with RSV "Aurora Australis", 999 drillhole measurements were made on three 500 meter long, $20 \mathrm{~m}$ spaced profiles in the sea ice, offering a unique dataset for ground truthing of the airborne EM data. For optimum validation data, a level ice floe with a prominent pressure ridge was chosen to lay out the profiles. Fig. 3 shows the thickness profiles illustrating level ice approximately half a metre thick, and a pressure ridge of thickness up to $5 \mathrm{~m}$. As a result of the footprint and the one dimensional processing algorithm, the maximum pressure ridge thickness is underestimated. The level ice thickness, however, is represented by the HEM data very close to the drilled estimates. 


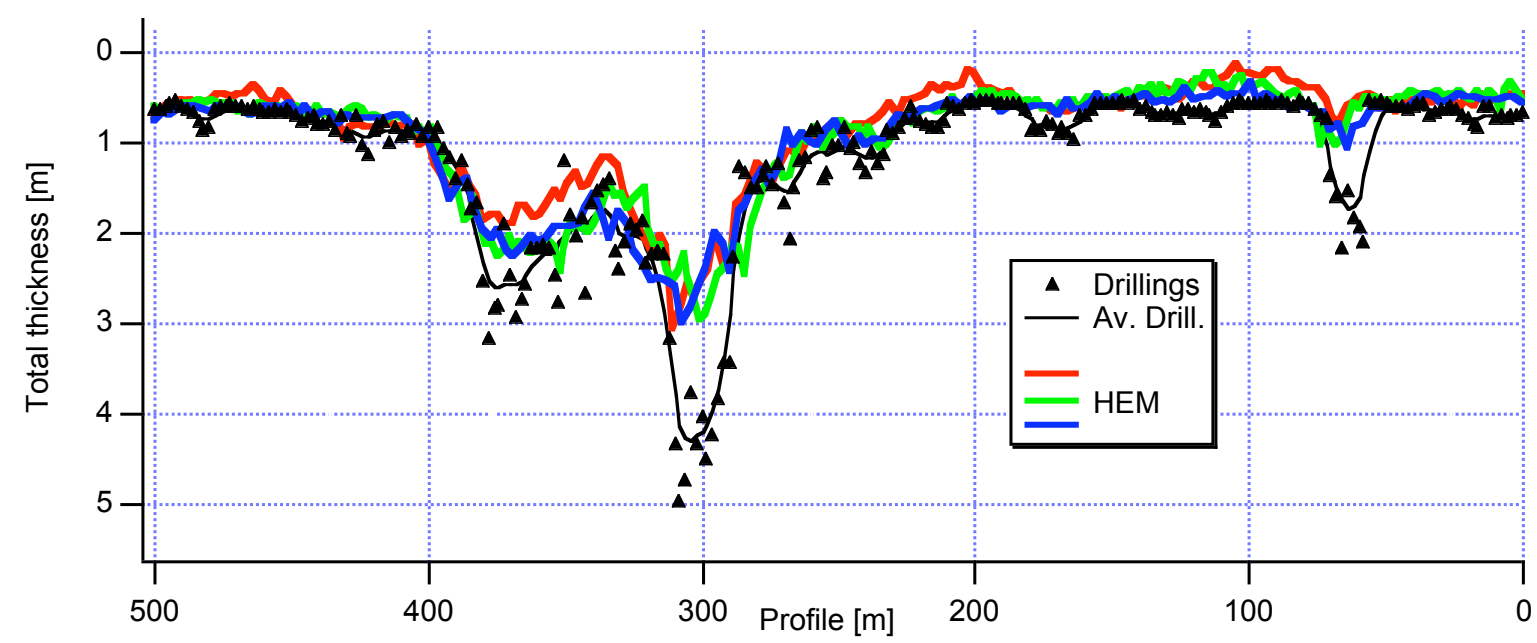

Figure 3: Sea ice thickness obtained by drilling and empirical HEM processing. Binomial smoothing of the drilling results is labeled as Av. Drill. Three thickness profiles for HEM arise from several overpasses over the drilled profile. Data from 3 of 10 flights along the profile are shown.

A key parameter in sea ice research is the ice thickness distribution and the consequently derived level ice thickness. Probability histograms of the thickness data in Fig. 3 are shown in Fig. 4. Data from the three HEM overpasses have been combined in one histogram and compared to the drilling information. As there is neither open water nor multi year ice present, the distributions show one dominant peak representing the level ice thickness. For both histogram resolutions 10 and $2 \mathrm{~cm}$ HEM and drilling data agree at 0.5 and $0.54 \mathrm{~m}$ thickness respectively.
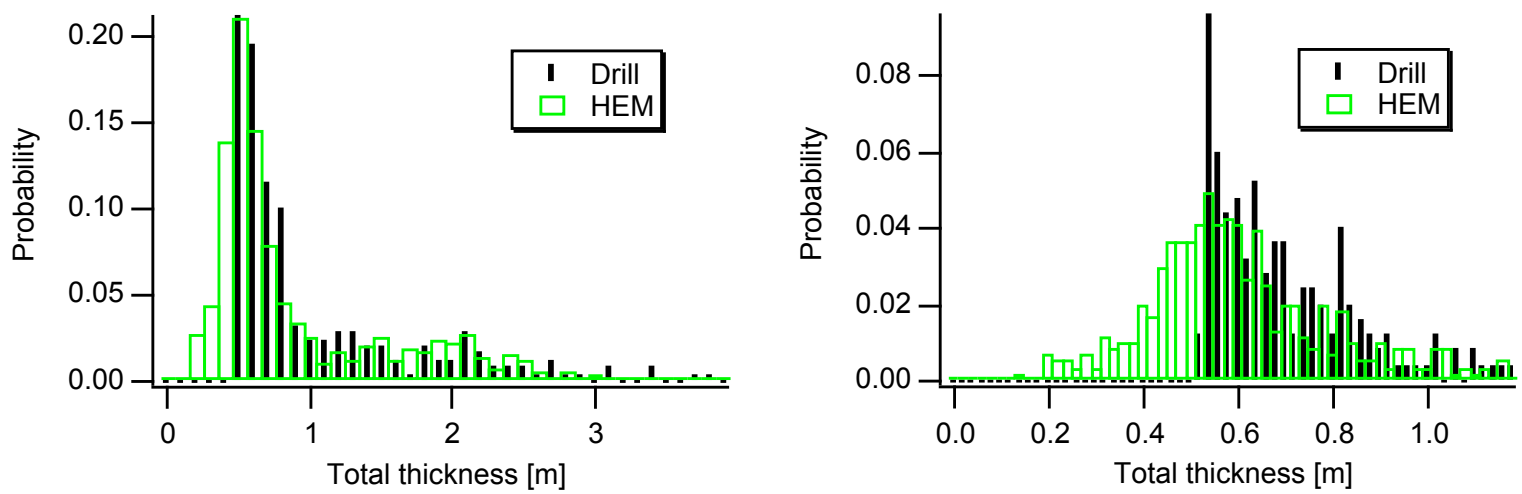

Figure 4: Ice thickness distribution determined form drillings and HEM data. (a) Histogram calculated with 10 $\mathrm{cm}$ bins. (b) $2 \mathrm{~cm}$ bins illustrating agreement of HEM and drilling regarding level ice thickness.

\section{Conclusion}

Field data and model studies have shown that, for the sea ice case, HEM data can be processed using a fairly simple empirical curve fitting approach. Preliminary comparisons of empirically-derived sea ice thicknesses with those from layered-earth inversion of the HEM data have shown very good agreement between the two methods. However, empirical interpretation can be performed in a fraction of the time required for formal inversion, and is comparatively insensitive to noise or other errors in the measured data.

\section{References}

Kovacs, A. and Holladay, J. S., 1990, Sea-ice thickness measurement using a small airborne electromagnetic sounding system: Geophysics, 55, 1327-1337. 